\title{
28 Research Soure \\ Caregiver Burden in Caregivers of Patients Receiving Hemodialysis: A Qualitative Study
}

\section{Sima Hejazi}

Shahid Beheshti University of Medical Sciences School of Nursing and Midwifery

https://orcid.org/0000-0002-8629-4091

Meimanat Hosseini ( $\nabla$ meimanathosseini@yahoo.com )

Shahid Beheshti University of Medical Sciences

Abbas Ebadi

Baqiyatallah University of Medical Sciences

Hamid AlaviMajd

Shahid Beheshti University of Medical Sciences School of Paramedical Sciences

\section{Research article}

Keywords: Caregivers, Caregiver Burden, Hemodialysis, Qualitative Research

Posted Date: October 6th, 2020

DOI: https://doi.org/10.21203/rs.3.rs-80671/v1

License: (c) (1) This work is licensed under a Creative Commons Attribution 4.0 International License. Read Full License 


\section{Abstract}

\section{Background}

Living with End Stage Kidney Disease and hemodialysis place too much burden on patients and their caregivers. The concept of caregiver burden describes a set of diverse caregiver experiences in providing care and requires a complex and multifaceted definition. Limited studies have looked at caregivers of patients receiving hemodialysis and the concept of caregiver burden from their perspective. This study aims to develop the concept of caregiver burden among family caregivers of patients receiving hemodialysis.

Method

This study was conducted using directed qualitative content analysis method. Twenty one family caregivers, patients, dialysis nurses, physicians, and social workers in teaching hospitals of Tehran and the Iranian Kidney Foundation, Tehran, Iran were enrolled using maximum variation purposive sampling method. Sampling was continued until data saturation was reached. The data collection method was indepth and semi-structured interviews. In order to analyze the data, the directed qualitative content analysis method was conducted based on the method proposed by Elo and Kyngas which was modified by Assaroudi et al.

Results

A total of 1162 codes, 63 subcategories, 18 generic categories, and 5 main categories were extracted. The concept of caregiver burden in family caregivers of patients receiving hemodialysis and its dimensions based on the dimensions of the Structural Model of Caregiver Burden Model were approved and another dimension titled a time-dependent burden added to it.

\section{Conclusions}

The caregiver burden experienced by caregivers of patients receiving hemodialysis has physical, psychological, emotional, spiritual, financial, social, and time-dependent dimensions.

\section{Background}

Caregiver burden is a multidimensional response to care-related physical, psychological, emotional, social, and financial stressors (1).

Living with End-Stage Kidney Disease (ESKD) and dialysis place too much burden on patients and their families (2). Family caregivers are individuals who take responsibility for meeting the major physical, emotional, financial, and social needs of the patient from hospitalization to providing care at home. The breadth of care required for a patient with ESKD causes the caregiver to be more vulnerable to emotional, physical, and psychological consequences, which are essentially greater in countries with more limited 
resources (3). The complications in caring for a patient receiving hemodialysis aggravate depression and reduce the quality of life of the caregiver (4). The burden on the caregiver directly affects the quality of care provided by them and may lead to inadequate care or even the abandonment of patient (5). Numerous studies have been conducted on issues related to caregivers of Alzheimer's patients; however, little is known about other diseases (6). Admittedly, caregivers and families of patients receiving hemodialysis have not been well understood, and what has been in the spotlight was primarily the patient receiving hemodialysis, not his or her caregiver. Limited studies have looked at the personal lives of caregivers of these patients (4). In one qualitative study, caregivers of patients receiving hemodialysis suffered from burden which formed four categories: care challenge, psychological vulnerabilities, the chronic nature of care and care in the shade. These categories had formed one theme: progressive exhaustion (7). In another qualitative study, three themes emerged from the experience of caregivers of these patients: constant struggle to learn, effort and adherence to the divine thread, and in disease's captivity (8). Demographic changes and therapeutic developments in chronic disorders may soon lead to an increase in the importance of informal care (1). It is also estimated that the total number of people with ESKD receiving renal replacement therapy will increase (9). Therefore, it is necessary to identify the concept of caregiver burden and its dimensions, and consequently, to make plans to determine this burden and implement measures to reduce it. Accordingly, in this study, based on the definition provided and the dimensions of caregiving burden in the 'Structural Model of the Caregiver Burden Model' proposed by Chou $(10,11)$, the concept of caregiver burden was developed. In this model, caregiver burden is considered as the caregiver's subjective perception of the burden increase in one of the physical, psychological, social, or financial levels during the care process.

\section{Methods}

\section{Aim}

The objective of this study was developing the concept of caregiver burden among family caregivers of patients receiving hemodialysis.

\section{Approach}

This study was conducted using a directed qualitative content analysis (DQCA) method. In case there is a previous theory or research findings about a phenomenon that are either incomplete or more research leads to providing additional descriptions about them, the researcher chooses the DQCA method (12). Since several studies had already been done on the concept of caregiver burden and specifically based on the definition and dimensions of caregiver burden in the Model proposed by Chou $(10,11)$, this method was used. The research setting was the waiting room in the hemodialysis wards of Tehran teaching hospitals as well as the Iranian Kidney Foundation, Iran.

\section{Participants}


Participants included family caregivers, patients, dialysis nurses, physicians, and social workers in teaching hospitals of Tehran and the Iranian Kidney Foundation. Inclusion criteria for family caregivers included the no known mental illness, the ability to speak Persian, and responsibility for direct patient care. Patients with ESKD receiving maintenance hemodialysis who were physically and mentally able to participate in the study and were over 18 years of age were also. Nurses must have at least one year of work experience in the hemodialysis unit. Social workers and specialists who had experience in working with patients receiving hemodialysis were also included.

\section{Data collection method}

In this study, a maximum variation purposive sampling method was used (maximum diversity in terms of gender, age, level of education, treatment duration, and socioeconomic class in the participants). Sampling was continued until data saturation was reached. The data collection method was semistructured interviews using a combination of the questions derived from the model and open-ended questions. Because the data collection method was semi-structured interview, we don't have questionnaire in this study. An interview guide was prepared and tested before the interview (see additional file 1). The interviews were recorded with the participants' permission. Before starting the interview, the purpose of study was explained. Participants' demographic characteristics were recorded. No interview was repeated, and no participant withdrew from the interview. A total of 21 interviews were conducted with 15 family caregivers, two patients, two dialysis nurses, a physician, and a social worker.

\section{Data analysis}

The DQCA method was conducted based on Elo and Kyngas (13) method which was modified by Assarroudi et al. (14), using the Unconstrained Matrix and MAXQDA software version 10 as follows:

Phase 1. Preparation

One of the researchers tried to acquire the necessary general skills. The interview guide was developed, and a pilot interview was conducted and analyzed by the research team. The interview transcriptions were considered as an analysis unit, and an attempt was made to become immersed in the data by repeatedly reviewing the interview transcriptions.

Phase 2 - Organizing

The formative categorization matrix was extracted. The theoretical definition of the main categories was determined. Coding rules were compiled. An initial pilot interview was coded, and the coding accuracy was checked. Afterward, the main analysis was conducted, the meaning units were extracted and summarized, and the primary codes were assigned to them. Subcategories and generic categorizations were formed. Subsequently, a relation was formed between the generic categories and the main categories.

Phase 3: Reporting 
Include the report of all stages of DQCA and findings of study.

\section{Trustworthiness}

To ensure the rigor of the study, the criteria of Credibility, Dependability, Transferability, and Confirmability (15) and Elo et al.'s checklist (16) were used and were presented below and in table 1 respectively (see additional file 2).

Table 1. Responses to the Elo, et al.'s checklist to improving trustworthiness 


Phase of Subcategory Response in present study
Content
Analysis

Preparation Data collection method

In accordance with the purpose of directed content analysis, semi-structured interviews with observation and field notes were used.

Since the researchers' goal was to develop a caregiver burden model, a semi-structured interview seems appropriate.

A combination of descriptive and semi-structured questions was used in the interview.

Two pilot interviews were conducted. By reviewing the audio file of the interviews and their transcriptions and answering questions such as "Have I controlled the interview?" "Have I asked very limited questions with closed-ended answers?" An attempt was made to evaluate the role of the researcher in the interview and to reach self-awareness and control in the next main interviews.

The pre-test of the categorization matrix was performed in a pilot study.

Sampling method

Maximum variation purposive sampling method was selected. The target characteristics and inclusion criteria were described. Details about the characteristics of research units were described in Tables in the research results. Sampling was done with different economic, social, and educational levels. Moreover, caregivers with different family relationships (partner, child, sister) of both sexes, with a separate or shared living place with the patient were also studied.

The main caregivers of hemodialysis patients, patients, and professional caregivers who were interested in relating issues and could reflect their experiences and mindsets well were used.

Specific inclusion criteria were set for family caregivers, professional caregivers, and patients.

An attempt was made to select suitable samples for the research by observing the compliance of the research samples with the inclusion criteria and the criteria related to the informant participant.

The sampling process continued until data saturation was reached. Furthermore, after transcription of the second interview, the process of data analysis began and continued simultaneously with sampling.

Selecting the unit of analysis

Organizing
Categorization and abstraction
The unit of analysis was the transcript of each interview.

The procedure for forming codes from meaning units, subcategories, and categories were described in the Tables.

By summarizing and merging similar categories and 
subcategories, an attempt was made to reduce the number of concepts.

Efforts were made to ensure that there was no overlap between categories.

Interpretation

The transcripts of all the interviews and the codes extracted from them were observed by the research team to minimize personal interpretation of the data.

By repeatedly reviewing the interview transcripts and the extracted codes and subcategories, an attempt was made to obtain a correct interpretation of the data.

The techniques of member check and peer check were used.

Representativeness The process of coding, the formation of subcategories, and categories were frequently reviewed and its process was under the supervision of the research team.

Reporting Reporting results

An attempt was made to report the process of forming the main categories from generic categories by compiling Tables.

Attempts were made to use participants' quotes in accordance with the subcategories and main categories.

Reporting the process of analysis

An attempt was made to fully describe the process of data analysis.

The accuracy and rigor of the data in this study were evaluated based on two sets of criteria.

Credibility: the codes of one interview were handed in to one of the participant and she verified codes (member check). Moreover, the codes of one interview were provided to a nursing Ph.D. student, and the accuracy of the coding was investigated (peer check). The results were also presented to the research team, and the items requiring modification were edited.

Dependability: Adequate explanation of the interview process was provided to the participants. In addition, detailed documentation of the data collection procedure was prepared to enable the external audit to assess it. All participants were asked the same question. Lastly, in the research report, participants' statements were quoted extensively.

Transferability: Attempts were made to providing an accurate report of the study method, findings and quotations.

Transferability: While using the field notes, the researcher's perception of the participant's speech was also restated to them for confirmation of its accuracy. 
The concept of caregiver burden in family caregivers of patients receiving hemodialysis and its dimensions based on the dimensions of Chou's Model were approved and developed. From 21 participants, 15 were main caregivers, whose the characteristics have been presented in Table 2 .

Table2. The characteristics of main participants

\begin{tabular}{|c|c|c|c|c|c|c|c|}
\hline $\begin{array}{l}\text { Living } \\
\text { With } \\
\text { patient }\end{array}$ & $\begin{array}{l}\text { Hemodialysis } \\
\text { duration (year) }\end{array}$ & Relationship & Education & $\begin{array}{l}\text { Marital } \\
\text { status }\end{array}$ & Gender & $\begin{array}{l}\text { Age } \\
\text { (year) }\end{array}$ & $\mathbf{N}$ \\
\hline No & 2 (mounth) & Daughter & Diploma & Married & Female & 45 & 1 \\
\hline Yes & 9 & Son & Academic & Single & Male & 55 & 2 \\
\hline Yes & 15 (mounth) & Daughter & Academic & Single & Female & 37 & 3 \\
\hline Yes & 7 (mounth) & Spouse & Academic & Married & Female & 67 & 4 \\
\hline No & 8 & Son & Academic & Divorced & Male & 42 & 5 \\
\hline Yes & 4 & Spouse & Academic & Married & Female & 66 & 6 \\
\hline Yes & 3 & Daughter & Academic & Single & Female & 34 & 7 \\
\hline Yes & 7 & Spouse & Diploma & Married & Female & 32 & 8 \\
\hline Yes & 2 & Spouse & Primary & Married & Female & 51 & 9 \\
\hline Yes & 2 & Spouse & Illiterate & Married & Female & 55 & 10 \\
\hline Yes & 4 & Spouse & Illiterate & Married & Female & 52 & 11 \\
\hline Yes & 3 & Spouse & Primary & Married & Male & 63 & 12 \\
\hline Yes & 3 & Sister & Academic & Married & Female & 30 & 13 \\
\hline No & 10 & Daughter & Academic & Married & Female & 43 & 14 \\
\hline Yes & 6 & Spouse & Diploma & Married & Female & 34 & 15 \\
\hline
\end{tabular}

Two patients, 69 and 40-year-old women, two female nurses with 4 and 15 years of work experience, one male physician and one female social worker were included. The longest and the shortest interviews lasted 105 and 13:03 minutes, respectively. A total of 1162 codes, 63 subcategories, 18 generic categories, and 5 main categories were extracted, which are shown in Table 3.

Table3. Generic and main categories 


\section{Generic categories}

Burden of high costs

Insufficient financial resources

Physical impact

Risking self-care

Stressful nature of illness and care

Psychological regression

Failure to achieve sexual-psychological comfort

Stress of patient behaviors

Limitations, difficulty and coercion

Ambiguity in the patient's condition

The pressure of the hospital space and the inadequacy of

its equipment

Threat to individual life and social role

Pressure from others

Burden of professional caregivers and officials

Prolonged treatment and permanent care

Time consuming patient affairs

Burden of responsibility

Various and time consuming treatments

\section{Main categories}

Financial burden

Physical burden

Psychological, emotional and spiritual burden
Social burden

Time-dependent burden

In the Chou's Model (11), caregiver burden includes physical, emotional, financial, and social burdens. In addition to confirming these four dimensions, findings added another dimension titled a time-dependent burden. The findings are described below.

\section{Category 1. Financial burden}

Family caregivers considered financial issues as one of the factors causing burden as a result of caring for their patients. This category included four subcategories as below:

High additional costs

In the process of caring for their patients, family caregivers had faced multiple high costs such as treating comorbid problems. 
"... when a person is on dialysis, contrary to what others call it free dialysis, it has very high costs. The side costs of their illness are high ..." (P3)

\section{Expensive medication}

One of the caregivers' problems was the high cost of medications.

"... What matters is the cost of medicines. The cost of medicine is very high for us..."(P1)

Insurance restrictions

“...Insurance only pays for Eprex and Venofer. Their medicines are mostly vitamins, like vitamin D. Insurance doesn't cover them..." (P7)

\section{Career risks of the patient as a breadwinner}

"...Actually, when a person who starts dialysis is the breadwinner of the family, for example, the father of a family, well, when he starts dialysis, three days a week, he has practically no ability to work..." (P21)

\section{Category 2: Physical burden}

Patients' weakness and inability along with complications of hemodialysis had caused physical problems such as hypotension, which required the caregiver to accompany the patient and, as a result, had placed a physical burden on him/her. Most patients receiving hemodialysis were elderly, which had exacerbated their inability. This category included subcategories as below:

The caregiver's new physical illness

"... We have some caregivers that most of them, after a period of time, suffer from an incurable illness, like gynecological and physical illnesses ..." (P21)

\section{Physical impact due to patient transfer}

Weakness and inability of patients due to dialysis complications and the presence of comorbidities had caused movement disorders and imbalance in them. This had caused the patient to become dependent on and need a companion for moving and mobility.

“... Because of moving him, for example, there's usually a lot of pressure on my back..."(P1)

“... This morning I tried so hard to get him out of the car, so my shoulder made a clicking sound, also my waist... he's tall, and it's hard for me ..." (P9)

The physical burden of attending the dialysis center

The old age of patient, weakness, and worries about the complications of dialysis had obliged the caregiver to accompany the patient to the dialysis center. Most caregivers were forced to stay in the 
hospital during dialysis.

"... well, this is a severe burnout that they have to spend eight hours a day in the hospital..."(P21)

\section{Dietary restrictions}

Most of the foods forbidden for patients were not provided by caregivers; as a result, caregivers were deprived of such foods.

"... We have a dialysis patient, and we try not to buy many things, such as bananas and things that are harmful to him, so he doesn't eat them..." (P14)

A threat to self-care

Involvement in care had caused caregivers to be unable to implement self-care and deal with their medical problems.

“... I don't care about myself. Since last December, I was supposed to go to the doctor and show my test results, but I couldn't. I myself have a thyroid problem. I must have a biopsy once a year. I just had it last year." (P6)

\section{Category 3: Psychological, emotional, and spiritual burden}

The process of caring for a patient receiving hemodialysis and its complexities cause psychological, emotional, and spiritual burden on the caregiver. This category included subcategories as below:

\section{Concerns about vascular access}

Continuing the hemodialysis and even the patient survival depends on the proper vascular access. Consequently, one of the worries of the caregivers was the malfunction of vascular access.

"... The only thing for these patients to survive is the right operation of the fistula and catheter. Many times we see both the patient and the caregiver are very worried that they may stop working ..." (P19) Depression and anxiety about the patient's threatening physical condition

The complexity of caring could cause symptoms of stress and depression in caregivers. Complications of dialysis and the illness cause instability in the patient's condition. These conditions cause panic and anxiety for the caregiver.

“... I'm just stressed and restless ..." (P14)

“... I'm just worried when it's his dialysis time. I don't want anything bad happens..."(P11) 
One of the concerns of caregivers was to supply medicines.

"... about their medicines, whether the medicine can be found, or not; that's the matter ..." (P19)

Concerns about not causing stress for others

One of the concerns of caregivers was to try not to cause a burden for others in the process of giving care.

"... It's not fair if I involve my children in this issue. They like to come ..." (P12)

Concerns about distance and transportation to the hospital

The caregiver was obliged to transport the patient to hemodialysis center, and the concern about finding the vehicle had caused them anxiety and stress.

“... We get very annoyed on the way. The hospital's too far ..." (P1)

Concerns about transplantation

Planning for renal transplantation was associated with various issues and caused stress.

“... Well, the transplantation itself is another matter to be successful or not. It's all scary. Transplantation is something else. These are all worries that bother you ..." (P8)

Mental involvement and low spirits

Caregivers complained of low spirits and mental involvement.

“... Well, your mind is always involved ..." (P1)

Feelings of despair, helplessness, and fatigue

The caregiver had witnessed how the patient was suffering, without being able to help him/her. This had caused an emotional burden and a feeling of despair and helplessness. In addition, the continuous and lifelong process of care had resulted in the caregiver's mental fatigue.

"... I can't do anything. You know, when someone coughs badly, you give him some water. They shouldn't even drink too much water. I mean, you really can't do anything ..." (P7)

“...I can't, anymore. Believe me, I'm fed up." (P9)

Sleep disorders

Caregivers had experienced sleep disorders due to the patient's particular condition and needs. 
“... At night, because he can't sleep, he expects us not to sleep either. He just calls us. He wants to see if we're awake or not..." (P1)

\section{A threat to sexual health}

Sexual dysfunction of patient puts a burden on the partner, who is also the primary caregiver of the patient. On the other hand, the caregivers' sexual health is also at risk due to the types of burdens they experience.

“... I've become a cold person because of the burdens on me. I don't have that feeling anymore..."(P8)

\section{The patient's lack of understanding of limitations}

ESKD and hemodialysis are accompanied by many limitations and deprivations for the patient. One of the issues that caregivers struggled with was the patient's unwillingness to accept these restrictions.

“... Ever since he gets sick, he likes to go out. He was the one who didn't usually go out. Now, he suggests the places that aren't possible to go at all, because they have stairs. For example, he likes to wander in stores...." (P9)

The burden of the patient not adhering to the diet

One of the challenges for caregivers was patients' non-adherence.

“... In these four years, whatever l've asked him to eat or not to eat, he hasn't listened. He's eaten what he's wanted ..." (P11)

\section{Patient misbehavior}

In some cases, caregivers were harassed and abused by their patients.

"...for example, I wanted to insert a catheter, but I was beaten. I was holding my head so that he wouldn't punch me in the face ..." (P6)

“... Now, sometimes, he starts swearing..." (P6)

Difficulty managing the disease

Managing patients' condition, and their needs and complications was difficult.

“... They can't manage at all anymore; then, everything is out of control. Too much pressure is on those poor people ..." (P18)

Difficulty coordinating life issues with hemodialysis 
The hemodialysis program determined the patient and caregiver's life plan. As a result, all issues had to be coordinated with that program, which was a difficult task and could be a source of the burden on the caregiver.

"... If we want to plan any program, we should coordinate it with our mother's dialysis schedule ..." (P1)

\section{Feeling limited}

The issues and complexities of care had caused a feeling of limitation in caregivers' minds.

“... well, you should be limited all the time..." (P7)

The obligation to ignore feelings

Caregivers were often forced to ignore their feelings under multiple burdens.

"... In fact, what I say, something else is in my heart. I mean, my appearance and my inside are two different things ..." (P3)

The obligation to tolerate the present condition

The caregiver felt compelled to provide care. Due to the patient's condition and the lack of other caregivers, he/she had to endure the difficult and stressful situation.

“... What can I do? I have to. Can I leave? No ..." (P11)

\section{Restrictions on religious practice}

Caregivers' involvement in providing care had refrained them from religious practices.

“... I can't do my own things. I can't even say a prayer, let alone other things..." (P)

Lack of education and the absence of adequate information

The lack of education, as well as inadequate information, had placed a strain on the caregiver.

“... I'm upset that why there's no one to teach us about what we should do for our patient...." (P6)

Worries about the future

Caregivers were concerned about the unknown future of their patients and their own condition.

“... I'm worried about the future. How long should he be dialyzed like this? ..." (P15)

Hospital atmosphere burden

Attendance in the hospital and experiencing its atmosphere had placed an emotional burden on them. 
"... The hospital also depresses. You sit here, just hear crying, screaming, and shouting. Well, it has a great effect on your spirit. This is the hospital environment even you have nothing to do except this; it affects your spirits..." (P7)

\section{Distrust of dialysis machines}

According to caregivers, most of the hemodialysis machines were out of date and did not operate properly. This had caused caregivers to distrust the efficacy of the dialysis.

"...Most of the patients and caregivers have knowledge about treatment; they complain that they feel hemodialysis is not as effective for them as it's mentioned in the sources ... the quality of dialysis is their concern...." (P20)

\section{Category 4: Social burden}

Caring for a patient receiving hemodialysis had placed various social burdens on the caregiver. Social burden in this study included generic categories as below:

\section{A threat to social life}

The caregiver was deprived of social activities and presence in the community and friends' company:

“... For example, I was invited to a few parties, but I didn't go. They were my husband's close relatives. I apologized on the phone ..." (P1)

\section{Changing the life process and family involvement}

Long-term caring for a chronic patient had changed the process of family life:

“... We are deleting a part of our lives ..." (P3)

“... Now, our life has become two parts; one part: hospital; another part: the house ..."(P3)

\section{A threat to the caregiver's family life}

Being responsible for caring for the patient had threatened the caregiver's personal and family life.

"... These are the problems that have involved me, also my wife..." (P13)

\section{Travel restrictions}

Caregivers, when planning for a trip, were concerned about dialysis for their patients, and the problems at the destination in terms of non-cooperation in allocating a dialysis bed or the lack of a dialysis bed had made it complicated for the family to travel, resulting in travel restrictions. 
“... Well, we can't travel at all during the holidays or busy days of the year. Because we are worried that we won't find an empty dialysis bed ..." (P15)

\section{A Threat to job status}

The need to accompany the patient to dialysis sessions had led the caregiver to be absent from work, which in many cases had led them to leave the job, lose a job, or retire early.

“...My job status. Well, you lose your job! ..." (P7)

Risking the possibility of marriage

Full-time care for the patient had caused the caregiver's endless involvement and had deprived them of the possibility of planning for marriage.

"... This issue has affected my choices. My family is my preference. I mean, their peace shouldn't be lost. So, I'm sacrificing myself ..." (P3)

The patient's weak role and the need for replacing it

ESKD and hemodialysis cause the patients to be engaged all the time and their role in the family is practically diminished. This shrinking presence of the patient in the family, along with the need to replace the role of the patient with the caregiver, had put a burden on the caregiver.

"... My husband has so much pain and worries about dialysis, so he gets involved unconsciously..." (P8)

“... I, somehow, compensate for my mom's duties ..." (P3)

To be pitied

The caregiver had felt distressed in contact with others and their reaction.

"...Well, what others say, for example, they say: "what are you doing now?" "Now, your husband is like this". This annoys you ..." (P8)

A feeling of not being understood

Caregivers had the feeling that others did not understand them.

“... Some people don't understand. They say: "why do you take this much burden of life? why do you do this? No, let him do it himself." Well, if he could, he did it himself. I get upset..."(P8)

Patient abandonment by others

The caregiver had felt abandoned by others, receiving no support from them. 
"... The problem is that his family doesn't support him at all ..." (P15)

\section{Stigma}

The caregiver had felt that hemodialysis was considered as a stigma and label by others.

“... You know, I'm single. Well, I'm not so sure yet, but I think a man who wants to marry me, or his family, even if they don't know what dialysis is, they may think they're getting a girl from a dialysis family, or I may be like this in the future..." (P3)

\section{Not being noticed and being abused by others}

Caregivers had felt that they were abused by others.

"... and I'm, a kind of, being abused. Others are taking advantage of me being single because I am supporting her ..." (P5)

Challenges with other caregivers

The presence of several caregivers and the complexity of the patient's condition had caused conflict between caregivers.

"... Let me tell you that, my mother and what happens to her is something, and convincing my brothers and family is something else ..." (P5)

The burden of other's expectations

The caregiver was exposed to the expectations of others, which in turn had created an added burden.

"... My children expect me, too. For example, they take their children here and ask me to take care of them ...." (P18)

\section{Dissatisfaction with the authorities}

Caregivers had experienced burden because they had not received adequate support.

"...You know, the government must provide an additional budget for patients with specific diseases, something like a support package or something that helps their medical expenses..."(P3)

The burden of not being understood by the medical system

Caregivers believed that the healthcare team did not understand them.

“... It means; they don't put themselves in our shoes to see what we do to help our patients..." (P3) 
Caregivers experienced distrust of the medical team.

“... I'm worried about this type of care..." (P7)

Category 5: The time-dependent burden

This is a newfound category that does not exist in the model proposed in this research. The caregiver was faced with multiple tasks, the patient's severe dependence, and time-consuming care, resulting in time constraints. Subcategories in this category included as below:

Understanding the need for a constant presence

The condition of the patient receiving hemodialysis required a constant, 24-hour attendance of the caregiver.

“... My mum needs us to be by her bed, day and night ...." (P2)

Constant involvement in care and a threat to privacy

The constant engagement in care had hindered the caregiver from spending their personal and private time.

“...We give them all our time ..." (P2)

The difficulty of prolonged treatment and care

A patient receiving hemodialysis needs lifelong care. This lengthy care process had caused a burden to the caregiver.

“... Look, every disease has a time. For example, heart surgery lasts for six months. But a dialysis patient needs care for the rest of his life ..." (P7)

The burden of doing the patient's activity of daily living

The patients' inability to perform their activity of daily living has placed their responsibility on the caregiver:

"...It's on me. Giving a bath is on me. I do his affairs. I shave his face. I dress him..."(P11)

The difficulty of caring for vascular access

Vascular access for a patient receiving hemodialysis requires special care. Due to the inability and the old age of the majority of the patients, caregivers were in charge of caring for vascular access.

“... That catheter itself needs care. When I give a bath ... I take sterile gauze every time and wash it with that. When I take it out, I spray on it; I dry it with a hairdryer..." (P6) 
Patient's severe dependence

Most caregivers stated that the patient had become dependent on them.

“... she calls every minute and asks: "where are you?" She's very dependent on me..."(P12)

\section{Difficulty monitoring medication intake}

Numerous drugs needed to be taken by the patient receiving hemodialysis. Patients are often unable to take their medication alone.

“... I have to give him the pills on time. They are all on me. He can't take pills at all if I'm not at home. He doesn't know what pill to take..." (P11)

The need to prepare a special diet and monitor adherence to it

The ESKD patient is required to adhere to strict dietary restrictions. The preparation of this diet was mainly the responsibility of the caregiver.

“... For example, my children prepare her food separately once a week..." (P12)

Losing time and difficulty of accompanying the patient to dialysis sessions

Patients receiving dialysis could not attend hemodialysis sessions alone due to disease complications and inability; as a result, caregiver had to accompany them to the hospital and stay there to return their patients home.

"... In general, the day of dialysis is somehow removed from the days of the week...."(P3)

Taking the responsibility of care alone

The caregiver felt he/ she had been abandoned in providing care.

“... I take care of them all by myself ..." (P6)

An increase in the burden of responsibility

An illness of a family member and limitations resulting from his/her weakness and vascular access had increased the responsibilities of the caregiver.

“... I go home from hospital, I just start to wash and cook, I should do all these ..." (P18)

Underlying and associated diseases: aggravating the situation

ESKD in most patients is mainly secondary to underlying diseases such as diabetes and hypertension. In addition, due to the fact that most of patients receiving hemodialysis were elderly, the underlying 
diseases were more common in them. As a result, in addition to managing ESKD problems, family caregivers had faced problems of underlying comorbidities.

"...Well, they have many more problems. Four days a week, she is on dialysis; the other two days, it's her heart disease, her anemia, her blood sugar and diabetes, her blood pressure, her triglyceride. We have all these problems ..." (P12)

Vascular access: problematic and time-consuming

Vascular access in patients receiving hemodialysis is accompanied by specific complications. The vascular access placement process was time-consuming. The need for hospitalization, multiple physician visits, and diagnostic tests had placed a burden on the caregiver.

"...At first, he was with a fistula, but it didn't work. There was no blood flow; his hand turned black. Then, they placed it on his chest; it became infected. They put it on the other side; it infected too. We've taken him to the hospital so many times..." (P11).

Involvement in a variety of treatments and associated problems

ESKD and dialysis treatment had caused complications, which had led to frequent hospitalization, the need for multiple and periodic visits by a physician, and the necessity of performing multiple diagnostic tests, whose responsibility had been placed on the caregiver.

“... My husband gets hospitalized a lot because of his problem. Just two months ago, he was hospitalized because of a cold ..." (P15).

\section{Discussion}

This study was carried out with the aim of developing the concept of caregiver burden in family caregivers of patients receiving hemodialysis, and one main category, i.e., the time-dependent burden was added to the previous four categories in the Chou's model $(10,11)$.

One of the issues raised in the present study was job-related issues. The caregiver's employment status was threatened due to his/her involvement in care. This issue had similarly been raised by caregivers in the study by Salehi Tali et al. (7). The need to attend the hemodialysis sessions with the patient, the need to hospitalize the patient, and the lack of supportive labor laws could cause the caregiver's career to be at risk.

Concern about the future was one of the factors creating the caregiver burden in this study. Family caregivers in the study by Rabiee et al. reported uncertainty about the future (8). The dependence of the patient's life on Renal Replacement Therapies (RRT) and the lifelong need for them were among the factors of concern about the future in these caregivers. One of the concerns of caregivers of patients with 
multiple sclerosis was, similarly, a concern about the future (17). It seems that the chronic and debilitating nature of multiple sclerosis can create similar concerns in caregivers.

One of the issues that caregivers addressed in the present study was sexual problems. In another study, one of the issues raised by caregivers of patients receiving hemodialysis was sexual function challenges, too(7). Patients with ESKD suffer from sexual dysfunction, which causes problems for their partners, who are often their primary caregivers. On the other hand, various psychological burdens on the caregiver might also affect their sexual desire.

Another source of a burden on caregivers in this study was the feeling of despair. Caregivers felt unable to deal with patients' conditions. Patients' caregivers in Cooper et al.'s study experienced similar feelings (18). The caregiver did not know what to do to improve the patient's condition. The chronic nature of the disease and its association with other diseases, as well as the old age of most patients, had complicated the patient's condition.

The high cost of patient care was another burden in the present study. In the study by Goodarzi et al., one of the challenges faced by caregivers of patients with the vegetative state was the ruinous costs (19). The chronic nature of ESKD and the characteristics of the patient with the vegetative state require multiple medical visits and treatments, preparation of special diet, provision of medical equipment, and frequent hospitalizations.

In the present study, one of the caregivers' issues was the difficulty of disease management. The dimensions of the patient's issues, which the caregiver was required to deal with, were so extensive. The results of the study by Goodarzi et al., similarly, led to the extraction of the main theme titled undertaking hard caring (19), which is parallel to the present study. The loneliness of the caregiver in providing care, which was one of the extracted subcategories in the present study, and the lack of a supportive social network, could make disease management more challenging.

Half of the caregivers of patients receiving hemodialysis studied by Intas et al. suffered from sleep disorders (20), as in the present study, one of the factors causing a burden on the caregivers was sleep disorders. Frequent awakenings and sleep disturbance of the patient due to the complications of the disease may cause sleep disturbance in the caregiver.

In the present study, caregivers experienced constant involvement in care that was a threat to their private life. The results of a study by (21) showed that partners caring for stroke patients had similarly experienced. In both hemodialysis and stroke patients, spending too much time providing care, multiple care tasks, and patient needs lead to constant caregiver involvement. Caregivers of dialysis patients in another study had; likewise, been limited in their lives due to the challenging and tough conditions they had endured (8).

One of the caregivers' psychological problems in the present study was depression and anxiety. The results of a systematic review in caregivers of cancer patients reported the high prevalence of depression 
and anxiety in them (22) that is consistent with our study. As a result of providing care, caregivers experience psychiatric problems (11). Studies have shown that caregivers' mental state is more affected than their physical condition (23).

In this study, an attempt was made to investigate the different groups of family caregivers by choosing the maximum variation sampling method. However, due to the focus of the study in Tehran, the capital of Iran, the concerns of caregivers living in small towns or villages might have been neglected.

\section{Conclusions}

The caregiver burden experienced by caregivers of patients receiving hemodialysis has physical, psychological, emotional, spiritual, financial, social, and time-dependent dimensions. On the one hand, caregivers suffer from self-care negligence, and on the other hand, the healthcare team does not provide adequate support for them, they are abandoned concerning care issues and experience financial and social burden, and as a result of providing care, they suffer from anxiety and depression. Consequently, caregivers, whose performances assure the patient's survival, are at a high level of vulnerability. The findings can be beneficial to identify the issues of caregivers of patients receiving hemodialysis and provide specific tools for measuring caregiver burden and to develop supportive and rehabilitation programs planned by nurses and social workers which can help to improve the condition of family caregivers.

\section{Abbreviations}

ESKD: End Stage Kidney Disease

DQCA: Directed Qualitative Content Analysis

RRT: Renal Replacement Therapy

\section{Declarations}

Ethical approval and consent to participate: Participants were assured that interviews would be kept confidential. Written informed consent was obtained. The present study is a part of Ph.D. dissertation which was approved by Shahid Beheshti University of Medical Sciences with the ethics code IR.SBMU.PHARMACY.REC.1398.99 and was sponsored by this university.

Consent for publication: Not applicable.

Availability of data and materials: The datasets used and analyzed during the current study are available from the corresponding author on reasonable request.

Competing interests: The authors declare that they have no competing interests. 
Funding: This study was supported by Shahid Beheshti University of Medical Sciences. The funder had no role in the design and writing the manuscript and will have not in data collection and analysis of data.

Authors' contributions: SSH, MH, AE and HAM led the conceptualization and design of the study. SSH collected data and SSH, MH, AE analyzed data. MH, AE and HAM substantively revised the study. SSH wrote the first draft and $M H, A E$ and HAM critically reviewed it and provided comments to improve the manuscript. SSH, MH, AE and HAM read and approved the final manuscript. SSH, MH, AE and HAM have agreed both to be personally accountable for the author's own contributions and to ensure that questions related to the accuracy or integrity of any part of the work, even ones in which the author was not personally involved, are appropriately investigated, resolved, and the resolution documented in the literature. All authors have read and approved the manuscript.

Acknowledgements: Not applicable.

\section{References}

1. Fekete C. Caregiver Burden. In: Wright JD, editor. International Encyclopedia of the Social \& Behavioral Sciences (Second Edition). Oxford: Elsevier; 2015. p. 135-8.

2. Hoang VL, Green T, Bonner A. Informal caregivers of people undergoing haemodialysis: Associations between activities and burden. 2019;45(3):151-8.

3. Oyegbile YO, Brysiewicz P. Exploring caregiver burden experienced by family caregivers of patients with End-Stage Renal Disease in Nigeria. International Journal of Africa Nursing Sciences. 2017;7:136-43.

4. Ebadi A, Sajadi SA, Moradian ST, Akbari R. Suspended Life Pattern: A Qualitative Study on Personal Life Among Family Caregivers of Hemodialysis Patients in Iran. International Quarterly of Community Health Education. 2018;38(4):225-32.

5. Sajadi SAM, Ebadi AP, Moradian ST. Quality of Life among Family Caregivers of Patients on Hemodialysis and its Relevant Factors: A Systematic Review. International journal of community based nursing and midwifery. 2017;5(3):206-18.

6. Faison KJ, Faria SH, Frank D. Caregivers of chronically ill elderly: perceived burden. J Community Health Nurs. 1999;16(4):243-53.

7. Salehitali S, Ahmadi F, Hasanpour Dehkordi A, Noorian K, Fereidooni-Moghadam M, Zarea K. Progressive exhaustion: A qualitative study on the experiences of Iranian family caregivers regarding patients undergoing hemodialysis. International Journal of Nursing Sciences. 2018;5(2):193-200.

8. Rabiei L, Eslami AA, Abedi H, Masoudi R, Sharifirad GR. Caring in an atmosphere of uncertainty: perspectives and experiences of caregivers of peoples undergoing haemodialysis in Iran. Scandinavian journal of caring sciences. 2016;30(3):594-601.

9. Liyanage T, Ninomiya T, Jha V, Neal B, Patrice HM, Okpechi I, et al. Worldwide access to treatment for end-stage kidney disease: a systematic review. Lancet (London, England). 2015;385(9981):1975-82. 
10. Chou KR, LaMontagne LL, Hepworth JT. Burden experienced by caregivers of relatives with dementia in Taiwan. Nursing research. 1999;48(4):206-14.

11. Chou KR. Caregiver burden: a concept analysis. Journal of pediatric nursing. 2000;15(6):398-407.

12. Hsieh HF, Shannon SE. Three approaches to qualitative content analysis. Qualitative health research. 2005;15(9):1277-88.

13. Elo $\mathrm{S}$, Kyngas $\mathrm{H}$. The qualitative content analysis process. Journal of advanced nursing. 2008;62(1):107-15.

14. Assarroudi A, Heshmati Nabavi F, Armat MR, Ebadi A, Vaismoradi M. Directed qualitative content analysis: the description and elaboration of its underpinning methods and data analysis process. Journal of Research in Nursing. 2018;23(1):42-55.

15. Lincoln YS, Guba EG. But is it rigorous? Trustworthiness and authenticity in naturalistic evaluation. New Directions for Program Evaluation. 1986;1986(30):73-84.

16. Elo S, Kääriäinen M, Kanste O, Pölkki T, Utriainen K, Kyngäs H. Qualitative Content Analysis:A Focus on Trustworthiness. SAGE Open. 2014;4(1):2158244014522633.

17. Masoudi R, Abedi $\mathrm{H}$, Abedi P, Mohammadianinejad SE. Experiences of iranian multiple sclerosis patients' and their caregivers' regarding care and treatment outcomes. Jundishapur journal of chronic disease care. 2014;3(1):21-31.

18. Cooper DL, Powe BD, Smith T. Social support provided by and strain experienced by AfricanAmerican cancer caregivers. Supportive care in cancer : official journal of the Multinational Association of Supportive Care in Cancer. 2013;21(10):2719-25.

19. Goudarzi F, Abedi H, Zarea K, Ahmadi F. Caring experiences and challenges of families with patients in vegetative state. skums-jcnm. 2015;3(4):65-79.

20. Intas G, Rokana V, Stergiannis P, Chalari E, Anagnostopoulos F. Burden and Sleeping Disorders of Family Caregivers of Hemodialysis Patients with Chronic Kidney Disease-End Stage: A CrossSectional Study. Advances in experimental medicine and biology. 2020;1196:33-40.

21. López-Espuela F, González-Gil T, Amarilla-Donoso J, Cordovilla-Guardia S, Portilla-Cuenca JC, Casado-Naranjo I. Critical points in the experience of spouse caregivers of patients who have suffered a stroke. A phenomenological interpretive study. PloS one. 2018;13(4):e0195190.

22. Geng HM, Chuang DM, Yang F, Yang Y, Liu WM, Liu LH, et al. Prevalence and determinants of depression in caregivers of cancer patients: A systematic review and meta-analysis. Medicine. 2018;97(39):e11863.

23. Yang $X$, Hao Y, George SM, Wang L. Factors associated with health-related quality of life among Chinese caregivers of the older adults living in the community: a cross-sectional study. Health and quality of life outcomes. 2012;10:143.

\section{Supplementary Files}

This is a list of supplementary files associated with this preprint. Click to download. 
- Additionalfile2.docx

- Additionalfile1.docx

Page 25/25 